\title{
MODELING MATERIAL FAILURE IN CONCRETE STRUCTURES UNDER CYCLIC ACTIONS
}

\author{
By Rui Faria, ${ }^{1}$ Javier Oliver, $^{2}$ and Miguel Cervera ${ }^{3}$
}

\begin{abstract}
A constitutive model devised for the analysis of concrete structures, and suitable for generic $2 \mathrm{D}$ or $3 \mathrm{D}$ applications, is presented and validated. For plain concrete a tension-compression distinguishing stress split is performed, and two scalar damage variables account for the degradation induced by the tensile and compressive stress components. As outcomes the model reproduces the stiffness recovery upon load reversal, and it captures the strength enhancement under multiaxial compression. Besides, the simple formulation as well as the extremely reduced number of parameters involved in the concrete model makes it quite suitable for the analysis of real structures, and constitutes a useful design tool. As regards to the nonlinear performance of the steel reinforcement, the explicit Giuffrè-Menegotto-Pinto model is adopted. Efficiency of the global model is illustrated via two seismic applications: one concerning an arch dam, and the other a six-floor reinforced concrete wall. The latter application is presented for validation purposes.
\end{abstract}

Keywords: concrete, stress split, scalar damages, steel, cyclic behavior, experimental validation

\footnotetext{
${ }^{1}$ Associate Professor, Faculdade de Engenharia da Universidade do Porto, Civil Engineering Dept., Rua Dr. Roberto Frias, 4200-465 Porto, Portugal, Phone: (+351).22.508.1950, Fax: $(+351) .22 .508 .1835$, E-mail: rfaria@fe.up.pt.

${ }^{2}$ Full Professor, E.T.S. Ingenieros de Caminos, Canales y Puertos, Technical University of Catalonia, C/ Gran Capitán s/n, Edificio C1, 08034 Barcelona, Spain.

${ }^{3}$ Full Professor, E.T.S. Ingenieros de Caminos, Canales y Puertos, Technical University of Catalonia, C/ Gran Capitán s/n, Edificio C1, 08034 Barcelona, Spain.
} 


\section{INTRODUCTION}

The dissimilar behavior exhibited by concrete under tension or compression is an essential feature when dealing with cyclic actions. This peculiarity of concrete's behavior, also exhibited by other geomaterials, is a consequence of the rather different strengths exhibited under tensile or compressive loading, the first one associated to significant fragility, responsible for visible cracking. Therefore, under cyclic loading tensile cracking is usually the first evidence of nonlinearity, and consequently important changes in stiffness are observed upon reversal of the sign of the external loading, as it occurs during earthquakes.

To cope with this unilateral effect the devised concrete constitutive model must be able, somehow, to distinguish tension from compression, an objective that at a macro level is usually accomplished by implementing a split of the strain or the stress tensors (Ortiz 1985,

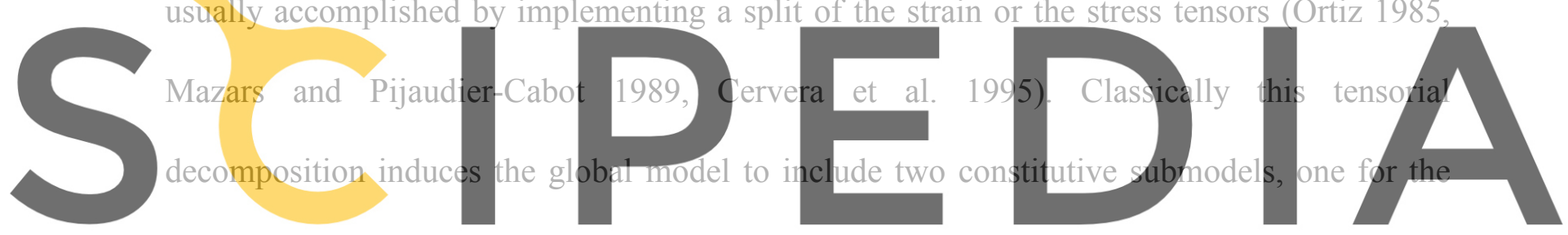

tensile and the other for the compressive tensor counterparts; typical associations of this kind

Register for free at https//www.scipedia.com to download the version without the watermark are the models which combine the 'smeared' or 'discrete crack approaches' with

plasticity-based yielding criteria. In spite of the considerable success of many of these submodel associations, an important drawback is well-known: the computational coding complexity, not only due to the duplication inherent to the tension and compression submodels, but also to the difficulty in dealing with combined tension-compression states of stress at each point (which one of the submodels should be fulfilled first, when a trial stress tensor violates both?). Furthermore, with such submodel associations seismic analyses of large structures become computationally almost prohibitive, regarding the many thousands of load steps and load reversals involved.

An approach that circumvents many of the above shortcomings, and becomes quite more 
attractive for civil engineering applications, is provided by the so-called scalar damage models, which are based on the assumption that with an appropriate set of internal variables the scalar damages - the nonlinear performances of concrete under tension and compression are manageable with an integrated strategy. In association to some split of the strain or the stress tensors, different scalar damage variables are introduced to cope with the dissimilar performances of concrete under tension and compression. Through appropriate evolution laws such damage variables, which range between 'zero' whilst material is elastic and 'one' (or some other limit) when material reaches collapse, provide a quite intuitive tool to monitor the internal progression of material degradation.

This paper concentrates on the problem of reproducing the nonlinear behavior of reinforced concrete $(\mathrm{RC})$ structures by adopting for the concrete part a constitutive model belonging to the fanily of scalar damage models. Quite refined approaches derived from,
damage mechanics are presently available (Ortiz 1985, Simo and Ju 1987, Chaboche 1988,
Lubliner et al. 1989, Mazars and Pijaudier-Cabot 1989, La Borderie et al. 1990, Ju 1990,

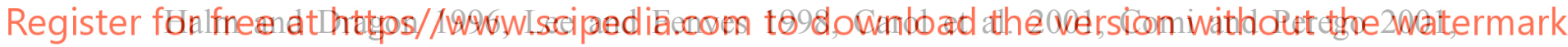

Hansen et al. 2001), some of them including tensorial damage variables, coping with material orthotropy or anisotropy, but the focus here is essentially to provide a numerical model suitable for engineering applications, keeping in good balance computational cost and efficiency. Accordingly, complex approaches other than the isotropy and scalar nature of the damage variables are declined, having in mind that the scope of this paper is to provide a constitutive model easily manageable for design purposes, and involving a reduced number of parameters. The model to be described for the concrete material is an upgraded version of the plastic viscous-damage one presented in Faria et al. (1998), where contributions from plastic deformations and viscous effects due to strain-rate dependency were discarded to allow for the intended aim of simplicity. In spite of its final intuitive format, the concrete model to be 
described here encompasses the dissimilar behavior under tension and compression, the stiffness recovery upon load reversal, it predicts the strength enhancement under $2 \mathrm{D}$ or $3 \mathrm{D}$ compression, and it is quite robust numerically. These features are accomplished by performing a split over the stress tensor obtained elastically, which includes the model in the so-called 'strain-driven' category, since the elastic stress tensor is computed explicitly on the basis of the strain tensor, the primary variable to be evaluated on standard finite element (FE) displacement-based codes. The dissimilar features of concrete's behavior under tension or compression are accomplished by assigning a separate scalar damage variable to each of the tensorial components of the elastic stress tensor.

Concerning the reinforcement, rebars are reproduced with a FE mesh constituted by 2-nodded truss elements. The nonlinear performance of each rebar is reproduced with the

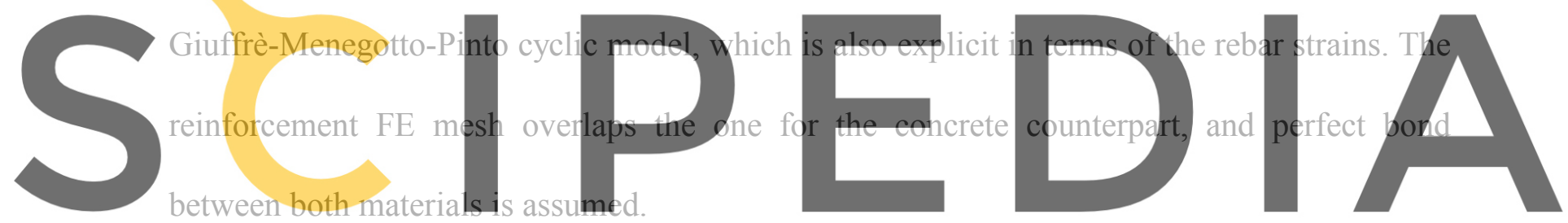

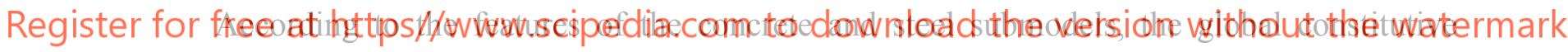

model that is presented for the analysis of RC structures is suitable for dealing with cyclic loading, with a straightforward implementation via a closed form algorithm, allowing for seismic analyses of really existing structures to become feasible. The efficiency of the global model is illustrated with two applications, namely a 3D plain concrete arch dam, and a six-floor 2D RC wall tested on a large-scale shaking table.

\section{BASIC CONCEPTS IN DAMAGE MECHANICS}

An essential idea in damage mechanics is that at each material point, and for an initial elemental area $A$ with a certain outwards normal, a reduction of the 'net' area occurs as an outcome of the propagation of micro-cracks and internal imperfections, induced by the 
external loading. On such elemental net area $A_{n}$ the actual stress is termed 'effective', here denoted by $\bar{\sigma}$, to be distinguished from the usual Cauchy $\sigma$ stress commonly adopted in structural applications. Following this interpretation $\bar{\sigma}$ is physically more representative than $\sigma$, as the latter corresponds to an averaged stress, idealized as acting on the initial area $A$, and not on the actual existing one $A_{n}$.

In combination with the effective stress concept one may also refer to the concept of scalar damage $d$, to be viewed under 1D conditions as the surface density of material defects existing at local level, that is, $d=1-A_{n} / A$. This damage variable evolves between 'zero' at the original elastic stage up to 'one' at material failure, and at any instant it quantifies the deterioration at a given point. Both the effective stress and the damage concepts are related, since equilibrium imposes that $\bar{\sigma} A_{n}=\sigma A$, and accordingly one has $\sigma=(1-d) \bar{\sigma}$.

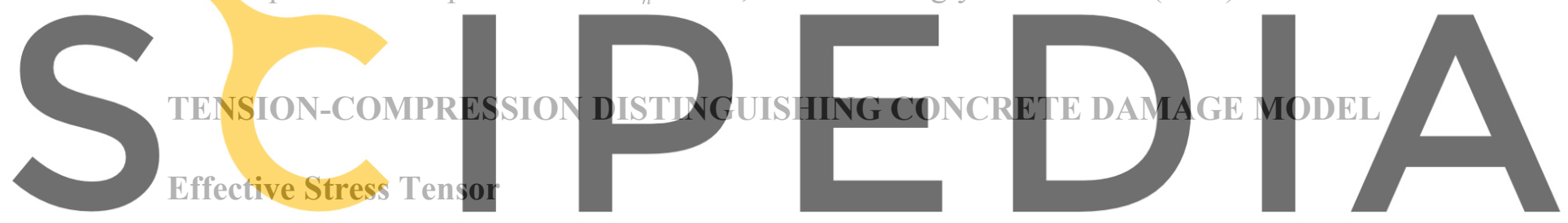

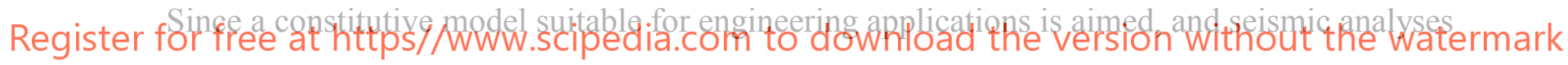

of large-scale concrete structures are envisaged, algorithmic efficiency is a subject of great concern. To account for this requisite, throughout the present chapter an explicit formulation in terms of the strain tensor is adopted, since $\varepsilon$ is the primary entity to be computed on standard FE codes. The well-known disadvantage of classical strain-based splits - namely the inability to cope with the strength enhancement in compression induced by the lateral confinement (see for instance Mazars and Pijaudier-Cabot 1989) -, is considerably reduced by adopting a stress tensor $\bar{\sigma}$ with an elastic definition as the basic entity within the model framework. This strategy preserves the advantages of a strain-driven formulation, since $\bar{\sigma}$ is directly computed in terms of $\boldsymbol{\varepsilon}$, and circumvents the drawbacks inherent to many implicit formulations based on the Cauchy stress tensor, where an iterative procedure inside the 
constitutive model is required to update the internal variables.

Therefore, and for the sake of simplicity, we will assume here the most simple strain-driven definition for the effective stress tensor $\bar{\sigma}=\left\{\bar{\sigma}_{11} \bar{\sigma}_{22} \bar{\sigma}_{33} \bar{\sigma}_{12} \bar{\sigma}_{13} \bar{\sigma}_{23}\right\}^{T}$, that is, $\overline{\boldsymbol{\sigma}}=\mathbf{D} \boldsymbol{\varepsilon}$, where $\mathbf{D}=$ the elastic constitutive tensor.

\section{Stress Split}

To account for the dissimilar performances of concrete under tension and compression, a split of the effective stress tensor $\overline{\boldsymbol{\sigma}}$ into tensile and compressive components $\overline{\boldsymbol{\sigma}}^{ \pm}$is introduced, and performed according to (Faria et al. 1998)

$$
\bar{\sigma}^{+}=\sum_{i}<\bar{\sigma}_{i}>\mathbf{p}_{i} \mathbf{p}_{i}^{T} \quad \bar{\sigma}^{-}=\bar{\sigma}-\bar{\sigma}^{+}
$$

where $<>=$ the Macaulay brackets (they retrieve the enclosed expression if positive, or zero
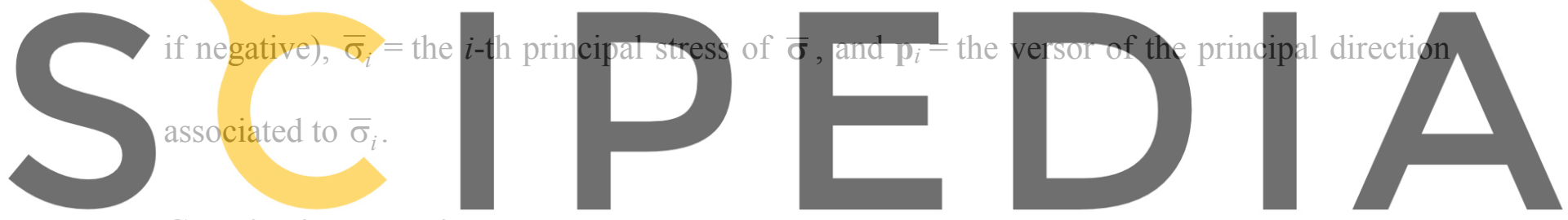

Constitutive Equation

Register for free at https//www.scipedia.com to download the version without the watermark

For a consistent derivation of a constitutive law a free energy potential with the form

$$
\psi\left(\varepsilon, d^{+}, d^{-}\right)=\left(1-d^{+}\right) \psi_{\mathrm{o}}^{+}(\boldsymbol{\varepsilon})+\left(1-d^{-}\right) \psi_{\mathrm{o}}^{-}(\boldsymbol{\varepsilon})
$$

is postulated, where $d^{+}$and $d^{-}$are scalar damage variables reproducing the tensile and compressive deteriorations (assumed as independent), and $\psi_{\mathrm{o}}^{ \pm}$are elastic free energies defined according to

$$
\psi_{\mathrm{o}}^{ \pm}(\boldsymbol{\varepsilon})=\frac{1}{2}\left(\overline{\boldsymbol{\sigma}}^{ \pm}\right)^{T} \mathbf{D}^{-1} \overline{\boldsymbol{\sigma}}=\frac{1}{2}\left(\overline{\boldsymbol{\sigma}}^{ \pm}\right)^{T} \boldsymbol{\varepsilon}
$$

(index \pm means tension or compression as appropriate). Note in these two equations that the constitutive model is intentionally written in terms of the effective stress tensor, a strain-driven entity as referred before. 
Having in mind that $\overline{\boldsymbol{\sigma}}^{ \pm}=\partial \psi_{\mathrm{o}}^{ \pm} / \partial \boldsymbol{\varepsilon}$ (see Faria et al. 1998 for details), the following form is obtained for the constitutive law

$$
\boldsymbol{\sigma}=\frac{\partial \psi}{\partial \boldsymbol{\varepsilon}}=\left(1-d^{+}\right) \overline{\boldsymbol{\sigma}}^{+}+\left(1-d^{-}\right) \overline{\boldsymbol{\sigma}}^{-}
$$

This relevant equation points out that the adopted split of the effective stress tensor leads also to a related dual split of $\sigma$ into tensile and compressive tensors $\sigma^{+}$and $\sigma^{-}$, that is,

$$
\sigma^{+}=\left(1-d^{+}\right) \bar{\sigma}^{+} \quad \sigma^{-}=\left(1-d^{-}\right) \bar{\sigma}^{-}
$$

Recalling conclusion (5), for general stress conditions (that is, $\bar{\sigma}^{+} \neq \mathbf{0}$ and $\bar{\sigma}^{-} \neq \mathbf{0}$ ) a non-null stress tensor $\boldsymbol{\sigma}$ is retrieved if $d^{+}=1$, provided that $d^{-} \neq 1$ (or conversely if $d^{-}=1$ and $\left.d^{+} \neq 1\right)$. Therefore, with the capability of activating the tensile and compressive damage variables independently the present damage model allows predicting the formation of
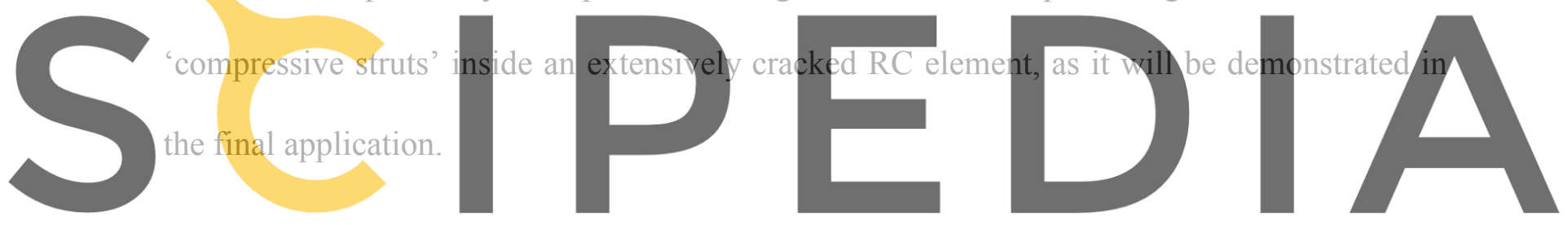

Damage Criteria

Register for free at https//www.scipedia.com to download the version without the watermark In the literature (Simo and Ju 1987, Mazars and Pijaudier-Cabot 1989, Oliver et al. 1990)

several definitions of tensorial norms and damage criteria are encountered. A crucial distinction between those norms and damage criteria concerns the basic entity on which they are based, and at least two families may be identified: (i) the strain-based ones, and (ii) the stress-based ones. Apparently this may be thought to reflect the different appraisals concerning the basic mechanisms which guide the initiation and progression of damage in concrete, particularly the one associated to cracking, where interpretations linking this phenomenon to lateral expansion (volume increase), or to tensile stresses, are commonly met. These interpretations depend mostly on the level under which the model approximation is introduced, since under a micro or mesoscopic level cracking in the cement paste is frequently 
attributed to tensile stresses that form due to bridging between the aggregates, whereas under a macroscopic standpoint, and for instance during a uniaxial compressive test, visible cracking is sometimes associated with positive lateral straining. Under a physical standpoint the approach to be adopted herein is more likely to be classified as stress-based, although computationally the norms and damage criteria will be handled as efficiently as strain-based entities.

Analogously to the 'equivalent strain' concept postulated in Simo and Ju (1987), positive scalar norms of the stress tensors and quite simple damage criteria will be adonted here to distinguish loading from unloading, and consequently to activate or deactivate the evolution of the internal variables $d^{ \pm}$. Such norms are termed equivalent stresses, denoted by the symbols $\tau^{+}$and $\tau^{-}$and computed according to

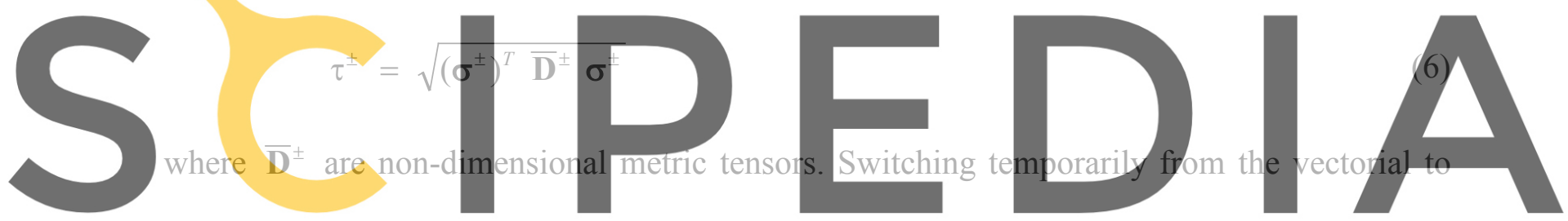

the second-order matrix notation of the stress tensors, the following definitions can be adopted Register for free at https//www.scipedia.com to download the version without the watermark for the metric tensors

$$
\overline{\mathbf{D}}^{ \pm}=\left(1+\gamma^{ \pm}\right) \mathbf{I}-\gamma^{ \pm} \mathbf{1} \otimes \mathbf{1}
$$

where $\mathbf{I}=$ the fourth- and $\mathbf{1}=$ the second-order identity tensors, and symbol $\otimes$ denotes the tensorial product. Parameters $\gamma^{ \pm}$are defined separately for tension and for compression, and are devised to reproduce the equibiaxial $\div$ uniaxial strength ratios typical in concrete.

Calling for the stress norms defined in (6), the following damage criteria in terms of the Cauchy stress tensors are introduced

$$
g^{ \pm}\left(\sigma^{ \pm}, q^{ \pm}\right)=\tau^{ \pm}-q^{ \pm} \leq 0
$$

where $q^{ \pm}$are current thresholds that control the size of the damage surfaces, therefore playing the role of hardening-like internal variables. 
Now, let us assume that the damage variables are computed in accordance to

$$
d^{ \pm}\left(r^{ \pm}\right)=1-\frac{q^{ \pm}\left(r^{ \pm}\right)}{r^{ \pm}}
$$

where the thresholds $q^{ \pm}$are positive functions of some internal variables $r^{ \pm}$, such that $\dot{r}^{ \pm} \geq 0$. Through substitution of (6) in (8), owing to the split of tensor $\sigma$ expressed in (5) and also to (9), it is worth noting that the criteria expressed in (8) are equivalent to

$$
\bar{g}^{ \pm}\left(\bar{\tau}^{ \pm}, r^{ \pm}\right)=\bar{\tau}^{ \pm}-r^{ \pm} \leq 0
$$

where

$$
\bar{\tau}^{ \pm}=\sqrt{\left(\bar{\sigma}^{ \pm}\right)^{T} \overline{\mathbf{D}}^{ \pm} \bar{\sigma}^{ \pm}}
$$

For practical applications the latter definitions provide a quite friendly format for the damage criteria, since they retain the scalar nature of the ones expressed in (8), and in spite of its

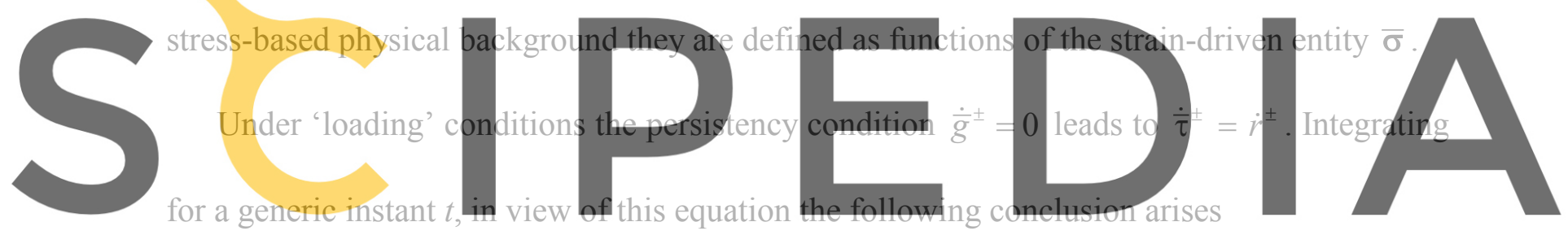

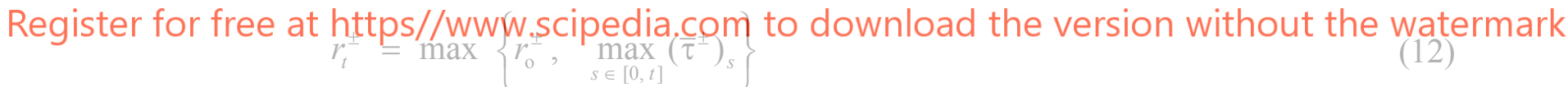

where $r_{\mathrm{o}}^{ \pm}$are the thresholds that bound the initial linear-elastic domain; according to (10) and (11), and since $\left.d^{ \pm}\right|_{t=0}=0$, it results $r_{\mathrm{o}}^{ \pm}=\mathrm{f}_{\mathrm{o}}^{ \pm}$, where $\mathrm{f}_{\mathrm{o}}^{ \pm}$denote the threshold stresses that define the onset of damages in $1 \mathrm{D}$ tension or compression. Assuming a ratio $\mathrm{f}_{\mathrm{o}}^{-} \div \mathrm{f}_{\mathrm{o}}^{+}=10$, Fig. $1 a$ reproduces the envelope of the $2 \mathrm{D}$ initial elastic domain inherent to the damage criteria expressed in (10): in pure tension a rounded Rankine-type criterion is obtained by setting $\gamma^{+}=0.0$, whereas in pure compression a equibiaxial $\div$ uniaxial strength ratio equal to 1.15 is obtained by adopting $\gamma^{-}=0.622$. The overall agreement of this envelope with the one inferred form the experimental results due to Kupfer et al. (1969) is fairly good. 
Regarding the non-negativeness of $\psi_{0}^{ \pm}$, from eqs (2) and (4) it can be inferred that for the dissipation inequality $\left(-\dot{\psi}+\sigma^{T} \dot{\boldsymbol{\varepsilon}} \geq 0\right)$ to be satisfied, that is,

$$
-\dot{\psi}+\sigma^{T} \dot{\boldsymbol{\varepsilon}}=\psi_{\mathrm{o}}^{+} \dot{d}^{+}+\psi_{\mathrm{o}}^{-} \dot{d}^{-} \geq 0
$$

it suffices that $\dot{d}^{ \pm} \geq 0$, a classical condition that rules the rate evolution of the damage variables. Since $\dot{r}^{ \pm} \geq 0$, according to (9) condition $\dot{d}^{ \pm} \geq 0$ is equivalent to

$$
\frac{q^{ \pm}}{r^{ \pm}} \geq \frac{\partial q^{ \pm}}{\partial r^{ \pm}}=\mathrm{H}^{ \pm}
$$

In view of the fact that $0 \leq d^{ \pm} \leq 1$, according to (9) inequality $q^{ \pm} / r^{ \pm} \leq 1$ has to be satisfied, which combined with (14) leads to the conclusion that $\mathrm{H}^{ \pm} \leq q^{ \pm} / r^{ \pm} \leq 1$. As depicted in Fig. $1 b$, this condition is trivially satisfied by the hardening/softening laws exhibited by the
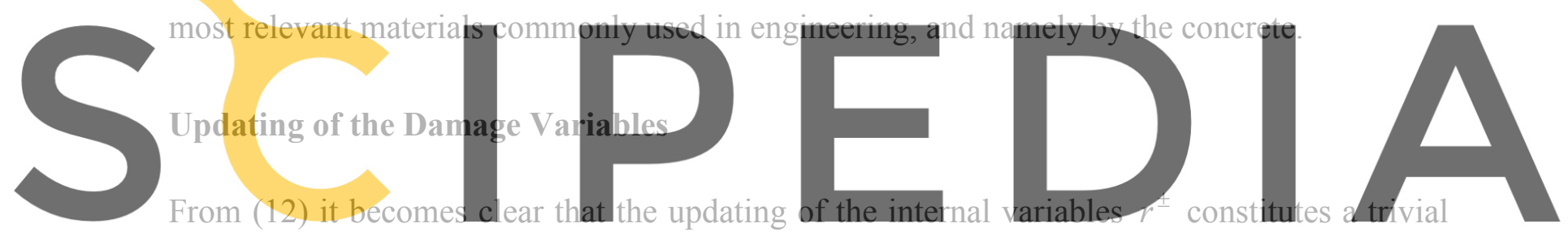

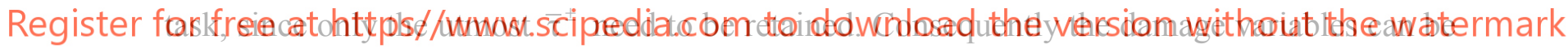

trivialiy updated too, because owing to (9) they are explicit functions of threshoids $r^{ \pm}$, provided that suitable formats are attributed to functions $q^{ \pm}\left(r^{ \pm}\right)$. In this paper the following evolution rules are adopted, which fulfill requirements (14):

$$
\begin{array}{ll}
q^{+}\left(r^{+}\right)=r_{\mathrm{o}}^{+} e^{A\left(1-r^{+} / r_{\mathrm{o}}^{+}\right)} & \text {if } r^{+} \geq r_{\mathrm{o}}^{+} \\
q^{-}\left(r^{-}\right)=r_{\mathrm{o}}^{-}(1-B)+r^{-} B e^{C\left(1-r^{-} / r_{\mathrm{o}}^{-}\right)} & \text {if } r^{-} \geq r_{\mathrm{o}}^{-}
\end{array}
$$

For a 1D tensile test eq. (15a) provides a softening branch that is asymptotic to the strain axis, and accordingly a finite area is retained between the stress-strain curve and the strain axis (see the envelope curve in Fig. 2a). As material softening engenders an ill-posed initial-boundary value problem, which leads to high sensitivity of the numerical solutions 
regarding the FE mesh refinement, as a consequence of strong tendency of deformations to localize on almost vanishing zones to form discrete cracks, a strategy for regularization needs to be introduced. The simplest one, also adopted here, consists in identifying the above referred finite area with the ratio $G / \ell$, where $G=$ the tensile fracture energy of concrete, and $\ell=$ the localization zone size. In Oliver (1989) a consistent definition for the characteristic length $\ell$ is defined, but regarding the purposes of the actual model we adopt here the following rough but simple definitions, based on the area $\Omega$ (or volume $\mathrm{V}$ ) of the current $2 \mathrm{D}$ (or 3D) finite element: $\ell=\sqrt{\Omega}$ or $\ell=\sqrt[3]{\mathrm{V}}$. Therefore, the unique parameter $A$ involved in (15a) is computed by identifying $G / \ell$ with the time integral of dissipation on a $1 \mathrm{D}$ tensile test, which after some mathematical handling renders (Oliver et al. 1990)

$$
A=\left(\frac{G E}{\ell\left(\mathrm{f}_{\mathrm{o}}^{+}\right)^{2}}-\frac{1}{2}\right)^{-1} \geq 0
$$

where $E=$ the Young's modulus.

Eq. (15b) allows reproducing the hardening in concrete under compression (Mazars and Pijaudier-Cabot 1989), as well as the softening that characterizes the post-peak behavior. Definition of the two parameters $B$ and $C$ is required, usually by imposing the numerical $\sigma-\varepsilon$ curve to pass in two selected points of a curve obtained from a 1D compressive test (if softening in compression is a subject of concern, a regularization scheme as referred for tension could also be adopted).

\section{Performance Under Cyclic Loading}

Fig. $2 a$ provides a pictorial description of the typical performance of the constitutive model during a 1D tension-compression cyclic test, where, for illustration purposes, the usual ratio between the compression and tensile strengths of concrete has been distorted. The tensile softening, as well as the hardening and subsequent softening observable under compression, 
are easily reproduced with the evolution laws expressed by eqs (15); the stiffness recovery when passing from tension to compression (or backwards) is also captured by the model.

\section{Algorithm}

Owing to the strain-driven formulation of the proposed model, its code implementation is quite straightforward, according to the following closed-form sequence of operations:

$$
\boldsymbol{\varepsilon} \Rightarrow \overline{\boldsymbol{\sigma}}=\mathbf{D} \boldsymbol{\varepsilon} \Rightarrow\left\{\begin{array}{l}
\overline{\boldsymbol{\sigma}}^{+} \Rightarrow \bar{\tau}^{+} \Rightarrow d^{+} \\
\overline{\boldsymbol{\sigma}}^{-} \Rightarrow \bar{\tau}^{-} \Rightarrow d^{-}
\end{array}\right\} \Rightarrow \boldsymbol{\sigma}=\left(1-d^{+}\right) \overline{\boldsymbol{\sigma}}^{+}+\left(1-d^{-}\right) \overline{\boldsymbol{\sigma}}^{-}
$$

\section{About the Definition of $\bar{\tau}^{-}$}

As depicted in Fig. $1 a$ the damage criteria (10), expressed in terms of the stress norms $\bar{\tau}^{ \pm}$ defined in (11), encompass an elastic domain that in pure tension and in pure compression is defined by ellipsoids. This presents some advantages, since through providing a unified format for the norms and the damage criteria associated to tension and to compression the mathematical handling of the corresponding expressions is considerably simplified. Nevertheless, under 3D compression the ellipsoid renders an excessively conservative envelope, like if a 'cap model' was activated for relatively low compressive stresses. Switching to another norm for the compressive stresses, namely the one

$$
\bar{\tau}^{-}=\sqrt{\sqrt{3}\left(K \bar{\sigma}_{\mathrm{oct}}^{-}+\bar{\tau}_{\mathrm{oct}}^{-}\right)}
$$

easily circumvents this limitation, as already proposed in Faria et al. (1998). In this format, directly inspired on the Drucker-Prager criterion, $\bar{\sigma}_{\text {oct }}^{-}$and $\bar{\tau}_{\text {oct }}^{-}$are the octahedral normal and shear stresses obtained from $\bar{\sigma}^{-}$; scalar $K$ controls the Drucker-Prager cone angle, and consequently it is calibrated to fit the experimental results in biaxial compression. Under plane stress conditions definition (18) performs almost identically to (11), leading to a bounding curve similar to the one depicted in Fig. 1a, but under 3D compression the open bounding surface inherent to the Drucker-Prager cone is more suitable for concrete, and 
adopted in the applications to be presented later. Nevertheless, the well-known insufficiency from the Drucker-Prager criterion consisting in that compressive stress states close to the hydrostatic axis remain elastic, irrespective to the stress intensity, is obviously a limitation also inherent to eq. (18), as it happens to many concrete devised plasticity-based models if not combined with 'caps'.

\section{Final Comments About the Concrete Model}

As remarked before, the elastic definition postulated for the effective stress tensor was greatly influenced by the goal of simplicity, and not by physical considerations. Obviously some limitations have to be expected as a result of neglecting the irreversible strains on the concrete model, namely the inability to control dilatancy under multiaxial stress conditions. The plastic-damage models described in Lubliner et al. (1989) and Lee and Fenves (1998) include a plastic strain tensor within the internal variables set, providing quite accurate predictions of the concrete's performance in several experimental tests, and accounting adequately for the dilatancy. A remark is yet made to the additional complexity of such refined approaches, both in terms of computational implementation and model calibration, which is contradictory to the straightforwardness intended for the present paper's model.

The option that was made to explicitly define the effective stress tensor as elastic engenders other limitations, namely the proficiency for tracking exactly the following features of concrete's behavior: (i) the energetic dissipation under cyclic loading, and (ii) the strength enhancement due to lateral confinement. Anyway, in several benchmark applications reported in Faria et al. (1998) the model predictions were not improved as expected when the plastic deformations were accounted for, in comparison to a pure damage model, yet with the latter providing numerical predictions accurate enough for the usual purposes of practical engineering applications. This observation has greatly influenced the option made to reduce the definition of $\bar{\sigma}$ to an elastic tensor. 


\section{STEEL CYCLIC MODEL}

In the applications of this paper the steel reinforcement is reproduced by discretizing each rebar via 2-nodded finite elements with axial behavior (truss elements), and thereafter superimposing such FE mesh to the one adopted for the plain concrete. An interface model accounting for bond-slip phenomena between concrete and steel would render a quite more detailed numerical representation, but model complexity would rise significantly. Besides, if for research or structural a posteriori analyses an adequate representation of slippage and rebar buckling phenomena are quite often needed, for applications involving design purposes they are usually discarded, among other reasons due to difficulties of calibration at the design stage, without experimental information. Accordingly, perfect bond between the concrete and the rebars is assumed hereinafter, and rebar buckling is neglected.

The nonlinear $\sigma-\varepsilon$ axial behavior of each rebar is simulated by the cyclic model reported in Menegotto and Pinto (1973), illustrated in Fig. 2b. As depicted transition curves are fitted between two asymptotes intersecting at point $\left(\varepsilon_{I}, \sigma_{I}\right)$, the latter changing its position according to the plastic incursion into tension or compression. The asymptotes have inclinations $E_{\mathrm{s}}$ and $E_{\mathrm{sh}}$ - the elastic and the hardening modulus of steel -, and the constitutive law is expressed by the explicit equation

$$
\sigma^{*}=\left\{\frac{E_{\mathrm{sh}}}{E_{\mathrm{s}}}+\frac{1-\frac{E_{\mathrm{sh}}}{E_{\mathrm{s}}}}{\left[1+\left(\varepsilon^{*}\right)^{B}\right]^{B^{-1}}}\right\} \varepsilon^{*}
$$

where

$$
\sigma^{*}=\frac{\sigma-\sigma_{r}}{\sigma_{I}-\sigma_{r}} \quad \varepsilon^{*}=\frac{\varepsilon-\varepsilon_{r}}{\varepsilon_{I}-\varepsilon_{r}} \quad B=\frac{-a_{1} \xi}{a_{2}+\xi}+a_{3} \quad \xi=\frac{\varepsilon_{r \max }-\varepsilon_{I}}{\varepsilon_{I}-\varepsilon_{r}}
$$

In these equations $\left(\varepsilon_{r}, \sigma_{r}\right)=$ the coordinates of the point where load reversal occurs, $\varepsilon_{r \text { max }}=$ the maximum $\varepsilon_{r}$ ever reached, and $a_{1}, a_{2}$ and $a_{3}$ are the parameters that fit the Bauschinger's 
effect observed on the $\sigma-\varepsilon$ rebar curves obtained experimentally.

\section{APPLICATIONS}

\section{Seismic Behavior of an Arch Dam}

The damage concept is naturally associated with physical deterioration, which makes it quite adequate to point out the post-elastic mechanisms activated when a structure is submitted to extreme load conditions. As an illustration, lets take the $110 \mathrm{~m}$ high Alto Lindoso arch dam (Portugal) reproduced in Fig. 3, where a FE mesh with 20-nodded bricks was adopted for the arch and foundation. This dam was analyzed under the $0.25 \mathrm{~g}$ earthquake also reproduced in Fig. 3. Without entering into dam engineering details, which are out of scope of the present paper (for further details see Cervera et al. 1995 and Faria 1998), here it is remarked that: (i) full reservoir condition was assumed; (ii) the foundation was assumed as elastic, and dissipation through radiation was accounted for by providing the boundaries with dampers endowing a transparency condition along the outwards normal (Faria 1998). Integration of the dynamic equations of motion was performed with the unconditionally stable and second-order accurate Hilber-Hughes-Taylor $\alpha$-method (Hilber et al. 1977), which provides a source of algorithmic dissipation quite useful for reducing spurious high-frequency oscillations (numerical noise). Concerning the viscous damping contribution, the following strategy was pursued: (i) whilst structural behavior remains elastic a Rayleigh-based stiffness proportional damping matrix is adopted, calibrated so as to provide $2 \%$ of the critical damping on the first mode, with a frequency of $2.5 \mathrm{~Hz}$; (ii) as tensile damages initiate and increase on a FE its damping matrix starts a continuous reduction process (in parallel to the stiffness matrix decay), keeping yet a residual damping matrix (of about $10 \%$ the original one) to compensate some insufficiency from the $\alpha$-method to bring down the numerical noise to an acceptable level (for further details see Faria et al. 2002). 
The basic properties and model parameters assumed for the concrete were the following: $E=30 \mathrm{GPa}, \quad$ Poisson's coefficient $=0.2, \quad \mathrm{f}_{\mathrm{o}}^{+}=2.5 \mathrm{MPa}, \quad G=200 \mathrm{~J} / \mathrm{m}^{2}, \quad \mathrm{f}_{\mathrm{o}}^{-}=7.5 \mathrm{MPa}$, $\mathrm{f}_{\mathrm{c}}^{-}=25 \mathrm{MPa}, K=0.171$, density $=2450 \mathrm{~kg} / \mathrm{m}^{3}$. The foundation was assumed with the same elastic properties of concrete, and with density $=2700 \mathrm{~kg} / \mathrm{m}^{3}$.

To get some insight on the nonlinear behavior of the Alto Lindoso dam during this moderately intense earthquake, Fig. 4 reproduces the evolution of the deformed configurations and the tensile damage distributions. No damages in compression were registered for this seismic intensity, and for clarity only the left half of the dam is represented. If one associates the occurrence of non-null values of $d^{+}$with concrete fractured regions, onset of cracking on the upstream heel and on the downstream face of the crest arch occurs at $t=1.57 \mathrm{~s}$, whilst the dam moves towards downstream. During the interval $1.57 \mathrm{~s}-1.84 \mathrm{~s}$ the crest deforms towards upstream, generating a curvature that promotes the spread of cracking on the downstream face. The ensuing deformation towards downstream $(t=2.06 \mathrm{~s})$ reverses the curvature on the center of the crest arch, engendering cracking on the upstream face, as indicated by wide regions with $d^{+}=1$ predicted at this elevation. Therefore, in practical applications the damage distributions provide an interesting tool for the analyst to interpret the structural changes induced by progression of nonlinearity: the spread of tensile damages depicted in Fig. 4 (and accordingly the associated structural induced stiffness decay) is easily recognized as responsible for the amplifying effect on the dam displacements, changing the fundamental vibration mode.

\section{Seismic Behavior of a RC Wall}

This application concerns the numerical simulation of the seismic behavior of a six-floor reinforced concrete wall, experimentally tested on a shaking table at the Centre d'Énergie Atomique (CEA), in France (see Combescure and Chaudat 2000 for details). Fig. 5 reproduces 
the meshes adopted for the concrete, discretized with 8-nodded plane stress FE (0.06 m thick), as well as for the steel reinforcement, simulated via 2-nodded truss elements. Four consecutive earthquakes were prescribed to the $\mathrm{RC}$ wall, according to the following sequence of intensities: $0.22 \mathrm{~g}, 1.35 \mathrm{~g}, 0.64 \mathrm{~g}$ and $1.0 \mathrm{~g}$. Even for the $0.22 \mathrm{~g}$ earthquake significant cracking was already induced in the wall, but in order to save space the results to be presented hereafter will refer only to the $1.35 \mathrm{~g}$ seism, reproduced in Fig. $6 a$.

Three different concrete domains A-B-C were considered in the wall (see Fig. 5), each of which with a particular 1D curve of the type depicted in Fig. $6 b$. Domain A concerns to the concrete standing outside the stirrups (unconfined concrete), whereas $\mathrm{B}$ and $\mathrm{C}$ refer to the confined concrete located within the core of the stirrups made up of $\phi 3$ diameters, with $0.02 \mathrm{~m}$ and $0.04 \mathrm{~m}$ spacings, respectively. Such distinction between the curves for the unconfined and confined concrete would be unnecessary under a 3D simulation, since the constitutive model would account for the lateral confinement provided by the stirrups. However, for the present simulation a $2 \mathrm{D}$ plane stress condition is being assumed, and consequently the concrete model cannot reproduce consistently the benefits provided by the confinement along the perpendicular to the plane of representation, since a null stress condition is enforced on such direction. Therefore, we adopt here the standard procedure that consists in attributing an increased compressive strength $\mathrm{f}_{\mathrm{cc}}^{-}$to the confined concrete depending on the confinement ratio $k$, with the latter being $k=1+A_{\mathrm{sw}} l_{\mathrm{w}} \mathrm{f}_{\mathrm{sy}} /\left(b_{\mathrm{c}} h_{\mathrm{c}} s \mathrm{f}_{\mathrm{c}}^{-}\right)$, where $A_{\mathrm{sw}}$ defines the cross sectional area of the stirrups, with perimeter $l_{\mathrm{w}}$, separation $s$, and yielding stress $\mathrm{f}_{\mathrm{sy}} ; b_{\mathrm{c}} \times h_{\mathrm{c}}$ designates the area of the concrete core effectively confined. Denoting by $\mathrm{f}_{\mathrm{c}}^{-}$and $\varepsilon_{\mathrm{c}}$ the compressive strength and strain for the unconfined concrete, the confinement effect may lead to the following increments on the concrete strength and peak strain (see notation in Fig. 6b): $\mathrm{f}_{\mathrm{cc}}^{-}=k \mathrm{f}_{\mathrm{c}}^{-}, \varepsilon_{\mathrm{cc}}=k^{2} \varepsilon_{\mathrm{c}}$ 
The following material properties were assumed invariable for the three concrete domains: $E=24.5 \mathrm{GPa}$, Poisson's coefficient $=0.2, \mathrm{f}_{\mathrm{o}}^{+}=3 \mathrm{MPa}, G=250 \mathrm{~J} / \mathrm{m}^{2}, \mathrm{f}_{\mathrm{c}}^{-}=39.6 \mathrm{MPa}, \varepsilon_{\mathrm{c}}=2.5 \%$, $K=0.171$, density $=2450 \mathrm{~kg} / \mathrm{m}^{3}$. In what concerns the confined concrete, domain B was simulated with $\mathrm{f}_{\mathrm{cc}}^{-}=54.7 \mathrm{MPa}$ and $\varepsilon_{\mathrm{cc}}=4.7 \%$, whereas in domain $\mathrm{C}_{\mathrm{cc}}^{-}=45.1 \mathrm{MPa}$ and $\varepsilon_{\mathrm{cc}}=3.2 \%$ were adopted. As for the steel reinforcement indicated in Fig. 5 the following material properties and model parameters were considered: $E_{\mathrm{s}}=200 \mathrm{GPa}, a_{1}=18.5, a_{2}=0.15$, $a_{3}=20.0$. Rebars $\phi 3$ and $\phi 4.5$ were simulated with $E_{\mathrm{sh}} / E_{\mathrm{s}}=0.0047, \mathrm{f}_{\mathrm{sy}}=563 \mathrm{MPa}$, and ultimate stress and strain with values $\mathrm{f}_{\mathrm{su}}=581 \mathrm{MPa}$ and $\varepsilon_{\mathrm{su}}=22 \%$; for rebars $\phi 6$ the steel properties were $E_{\mathrm{sh}} / E_{\mathrm{s}}=0.0052, \mathrm{f}_{\mathrm{sy}}=593 \mathrm{MPa}, \mathrm{f}_{\mathrm{su}}=625 \mathrm{MPa}, \varepsilon_{\mathrm{su}}=34 \%$, and finally for rebars $\phi 6 E_{\mathrm{sh}} / E_{\mathrm{s}}=0.0038, \mathrm{f}_{\mathrm{sy}}=486 \mathrm{MPa}, \mathrm{f}_{\mathrm{su}}=587 \mathrm{MPa}$ and $\varepsilon_{\mathrm{su}}=168 \%$ were adopted.

During the experimental test the intense $1.35 \mathrm{~g}$ earthquake induced important damages on the RC wall, associated to a quick progression of cracking and exploitation of large plastic deformations on the longitudinal rebars. The numerical predictions from the proposed model (obtained with the $\alpha$-method and the same strategy concerning the viscous damping referred for the previous application) are compared with the experimental results in Fig. 7, both in terms of the relative horizontal displacement registered at level 5, as well as in terms of the bending moment registered close to the footing (level 1). A good overall agreement between the model predictions and the test results was obtained, with the amplitudes, the frequencies and also the phases exhibiting acceptable deviations. The comparison performed in Fig. 8 concerns the moment-displacement diagrams obtained numerically and during the test campaign: it can be observed that the proposed model predicted fairly well the global performance of the RC wall, namely in what concerns the energetic dissipation registered experimentally. Finally, Fig. 9 reproduces a set of results obtained numerically at $t=4 \mathrm{~s}$, when the displacement registered on the top of the wall reaches the maximum. In Fig. $9 a$ the 
concrete tensile damages are superposed to the deformed configuration, and a comparison is possible with the crack pattern obtained experimentally, and reproduced in Fig. $9 b$ : the distribution of the tensile damages occurs at the same location where important cracks were registered during the test (in Fig. $9 b$ the cracks marked as "Before the test" were induced by the construction process - accordingly, they should be considered as spurious for the intended comparison). Defining by $\varepsilon_{\mathrm{p}, \max }$ the maximum post-yielding strain registered on the rightmost longitudinal $\phi 8$ (see Fig. 5), Fig. $9 c$ provides an insight on the incursion into yielding for that rebar: plastic straining concentrates mostly close to the wall's footing, associated to the formation of a main crack visible in Fig. $9 b$. It is also possible to observe that incursion into yielding extends approximately from the base up to level 3, a result in agreement to what is reported in the CEA report (Combescure and Chaudat 2000). Fig. $9 d$ reproduces the compressive stresses $\sigma^{-}$obtained with the proposed model, and puts into evidence the formation of inclined struts linking the compressed flange with the tie associated to the longitudinal reinforcement, that is, the materialization of the 'strut-and-tie' ultimate strength mechanism typical in RC structures.

\section{CONCLUSIONS}

This paper presents a constitutive model with an easily integrable format, devised for practical applications on large-scale RC structures, including seismic analyses. The model is capable of dealing with 2D and 3D structures, being constituted by two submodels: one concerning the concrete material, based on continuum damage mechanics, and one which deals with the reinforcement, based on the Giuffrè-Menegotto-Pinto explicit formulation.

Regarding the concrete submodel, an effective stress tensor is selected for supporting the formulation. This stress tensor, computed elastically, is split into tensile and compressive tensor components, each of which associated to an independent scalar damage variable. It 
allows for capturing the stiffness recovery effect upon load reversal, a feature of primary importance for seismic analysis. Owing to the strain-based formulation adopted throughout a closed-form and highly efficient integration algorithm is obtained. The submodel for the reinforcement is implemented on 2-nodded truss elements, to reproduce rebars made up of dissimilar steel grades.

With the purpose of keeping the global model easily manageable by practical engineers engaged with the analysis and design of plates, shells and full 3D structures, a reduced number of parameters is involved in both submodels. Among the possible outputs from the model, the plotting of the distributions of the tensile and compressive damages is an attractive tool to identify the structural ultimate strength mechanisms and critical points.

Two numerical applications were presented, illustrating the adequacy of the global constitutive model to reproduce the seismic behavior of an arch dam, as well as the earthquake performance of a reinforced concrete wall tested on a shaking table.

\section{ACKNOWLEDGEMENTS}

Research partially supported by the Training and Mobility of Researchers Programme, Access to Large Installations, under contract ERBFMGECT950062 “Access to supercomputing facilities for european researchers" established between The European Community and CESCA-CEPBA.

Financial support from the Spanish Ministry of Science and Technology, through grant MAT2001-3863-C03-03, and from the Catalan Government Research Department, through the CIRIT grant 2001-SGR 00262, is also gratefully acknowledged.

\section{APPENDIX I. REFERENCES}

Carol, I., Rizzi, E., and Willam, K. (2001). "On the formulation of anisotropic elastic degradation. II: generalized pseudo-Rankine model for tensile damage.” Int. J. Solids and 
Structures 38(4), 519-546.

Cervera, M., Oliver, J., and Faria, R. (1995). "Seismic evaluation of concrete dams via continuum damage models." Earth. Engrg. Struct. Dyn. 24, 1225-1245.

Chaboche, J. (1988). “Continuum damage mechanics. Part II: damage growth, crack initiation, and crack growth.” ASME J. Appl. Mech. 55, 65-72.

Combescure, D., and Chaudat, T. (2000). "Icons european program seismic tests on RC bearing walls: CAMUS 3 specimen.” Rep. SEMT/EMSI/RT/00-014A, CEA, France.

Comi, C., and Perego, U. (2001). "Fracture energy based bi-dissipative damage model for concrete.” Int. J. Solids and Structures 38(36-37), 6427-6454.

Faria, R., Vila Pouca, N., and Delgado, R. (2002). "Seismic behaviour of a R/C wall: numerical simulation and experimental validation.” J. Earth. Engrg. 6(4), 473-498.

Faria, R. (1998). "Seismic behaviour of concrete dams and its interaction with the rock

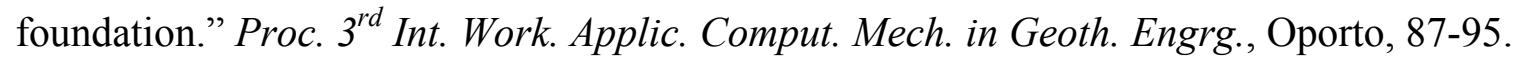

Faria, R., Oliver, J., and Cervera, M. (1998). “A strain-based plastic viscous-damage model for massive concrete structures.” Int. J. Solids and Structures 35(14), 1533-1558.

Halm, D., and Dragon, A. (1996). "A model of anisotropic damage mesocrack growth: unilateral effect.” Int. J. Damage Mechanics 5, 384-402.

Hansen, E., Willam, K., and Carol, I. (2001). "A two-surface anisotropic damage/plasticity model for plain concrete." Proc. FraMCoS-4 Conference, Cachan.

Hilber, H.M., Hughes, T.J., and Taylor, R.L. (1977). “Improved numerical dissipation for time integration algorithms in structural dynamics.” Earth. Engrg. Struct. Dyn. 5, 283-292.

Ju, J.W. (1990). "Isotropic and anisotropic damage variables in continuum damage mechanics.” ASCE J. Engrg. Mech. 116(12) 2764-2770.

Kupfer, H., Hilsdorf, H., and Rusch, H. (1969). "Behavior of concrete under biaxial stresses." J. Am. Conc. Inst. 66(8), 656-666. 
La Borderie, C., Berthaud, Y., and Pijaudier-Cabot, G. (1990). "Crack closure effects in continuum damage mechanics. Numerical implementation.” Proc. 2nd Int. Conf. Comp. Aided Analysis Design Conc. Structures, Zell am See, 975-986.

Lee, J., and Fenves, G.L. (1998). "Plastic-damage model for cyclic loading of concrete structures.” ASCE J. Engrg. Mech. 124(8), 892-900.

Lubliner, J., Oliver, J., Oller, S., and Oñate, E. (1989). “A plastic-damage model for concrete." Int. J. Solids and Structures 25(3), 299-326.

Mazars, J., and Pijaudier-Cabot, G. (1989). "Continuum damage theory. Application to concrete.” ASCE J. Engrg. Mech. 115(2), 345-365.

Menegotto, M., and Pinto, P. (1973). "Method of analysis for cyclically loaded reinforced concrete plane frames including changes in geometry and non-elastic behavior of elements under combined normal force and bending." IABSE Symp. Resist. Ultimate Deform. of Struct. Acted on by Well-Defined Repeated Loads, Final Report, Lisbon.

Oliver, J. (1989). “A consistent characteristic length for smeared cracking models." Int. J. Num. Meth. Engrg. 28, 461-474.

Oliver, J., Cervera, M., Oller, S., and Lubliner, J. (1990). "Isotropic damage models and smeared crack analysis of concrete.", Proc. 2nd Int. Conf. Comp. Aided Analysis Design Conc. Structures, Zell am See, 945-957.

Ortiz, M. (1985). “A constitutive theory for the inelastic behaviour of concrete." Mech. of Materials 4, 67-93.

Simo, J., and Ju, J. (1987). "Strain- and stress-based continuum damage models. I: formulation.” Int. J. Solids Structures 23(7), 821-840. 


\section{APPENDIX II. NOTATION}

The following symbols are used in this paper:

$a_{1}, a_{2}, a_{3}=$ parameters that control the steel Bauschinger's effect;

D, $\overline{\mathbf{D}}=$ elastic constitutive tensor and non-dimensional metric tensor;

$d \quad=\quad$ scalar damage variable;

$E \quad=\quad$ Young's modulus of concrete;

$E_{\mathrm{s}}, E_{\mathrm{sh}}=$ elastic and hardening modulus of steel;

$\mathrm{f}_{\mathrm{o}}=$ stress for the onset of nonlinearity under 1D loading;

$\mathrm{f}_{\mathrm{c}}^{-}, \mathrm{f}_{\mathrm{cc}}^{-}=$compressive strength of unconfined and confined concrete;

$\mathrm{f}_{\mathrm{sy}} \quad=\quad$ rebar yielding stress;

$G \quad=\quad$ tensile fracture energy in concrete;

$g, \bar{g} \quad=\quad$ damage criteria;

$\mathbf{I}, \mathbf{1}=$ fourth- and second-order identity tensors;

$K=$ parameter that controls the Drucker-Prager cone angle;

$q, r=$ internal variables that control the size of the damage surfaces;

$\boldsymbol{\varepsilon}=$ strain tensor

$\sigma, \bar{\sigma}=$ Cauchy and effective stress tensors;

$\gamma=$ parameter for the definition of $\overline{\mathbf{D}}$;

$\tau, \bar{\tau}=$ equivalent stress norms computed for the $\sigma$ and $\bar{\sigma}$ stress tensors;

$\psi \quad=\quad$ free energy.

\section{Superscripts}

,$+-\quad=\quad$ entity (or component) associated to tension or compression;

$\pm \quad=$ tension or compression, as appropriate;

\section{Subscripts}

o $\quad=$ elastic entity; value at the onset of nonlinearity. 


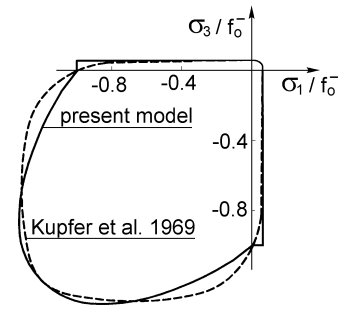

a)

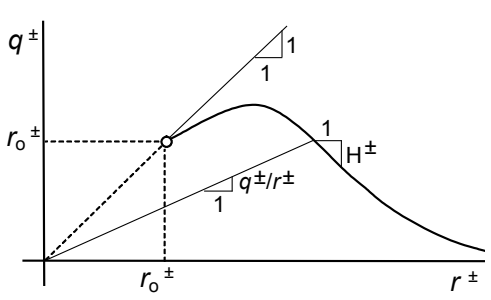

b)

FIG. 1. a) 2D Elastic Domain; b) Hardening/Softening Condition 


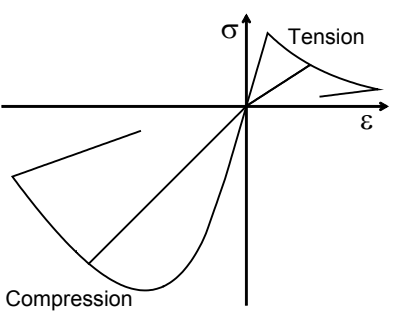

a)

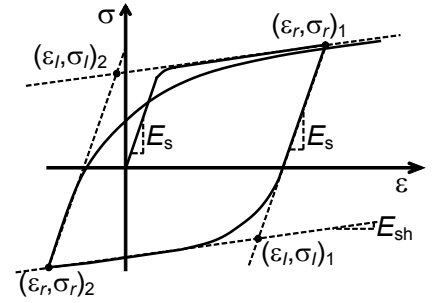

b)

FIG. 2. 1D Cyclic Performances of: $a$ ) Concrete; b) Steel Submodels 


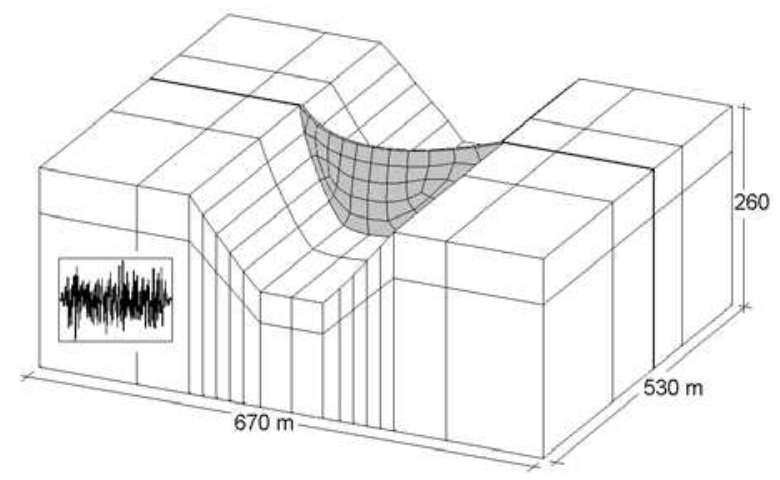

FIG. 3. Alto Lindoso Arch Dam 


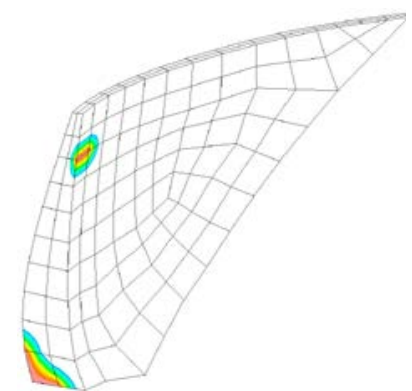

$t=1.57 \mathrm{~s}$

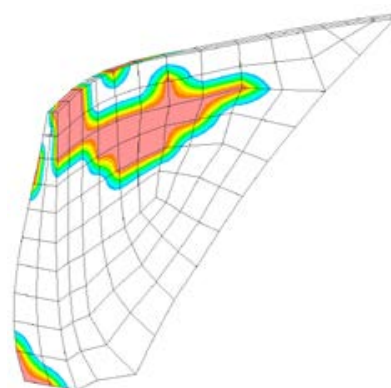

$t=1.73 \mathrm{~s}$

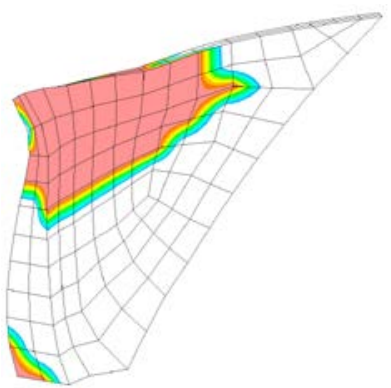

$t=1.84 \mathrm{~s}$

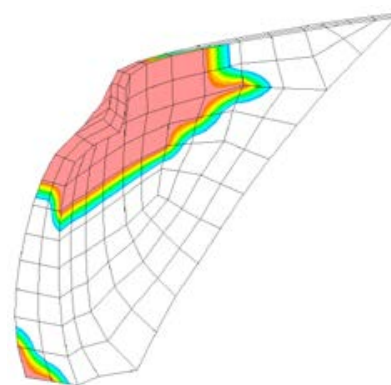

$t=2.06 \mathrm{~s}$

FIG. 4. Deformation and $d^{+}$During a $0.25 \mathrm{~g}$ Earthquake

$$
\left(d^{+}=1 \text { in gray; }=0 \text { in white }\right)
$$




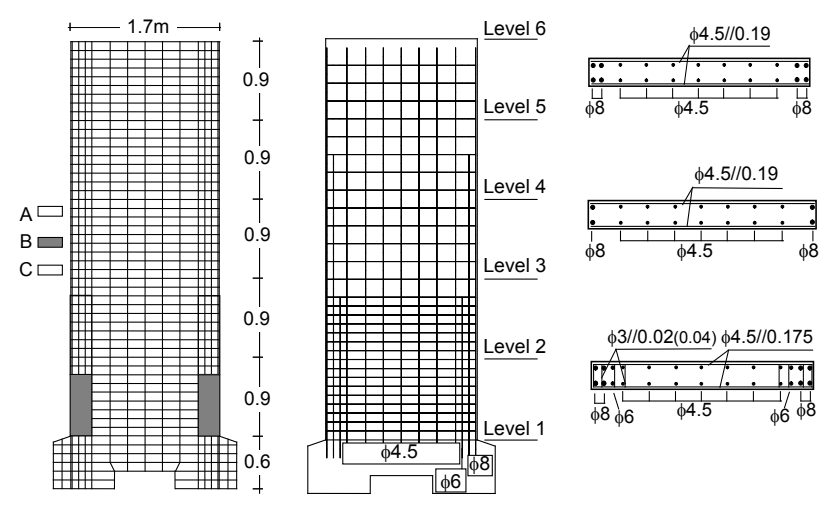

FIG. 5. FE Meshes and Geometrical Data for the RC Wall 


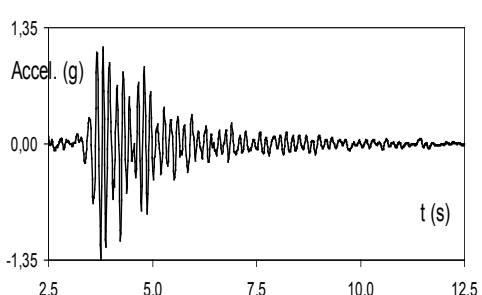

a)

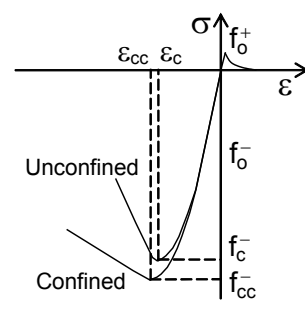

b)

FIG. 6. a) 1.35g Accelerogram; b) Confined and Unconfined Concrete 

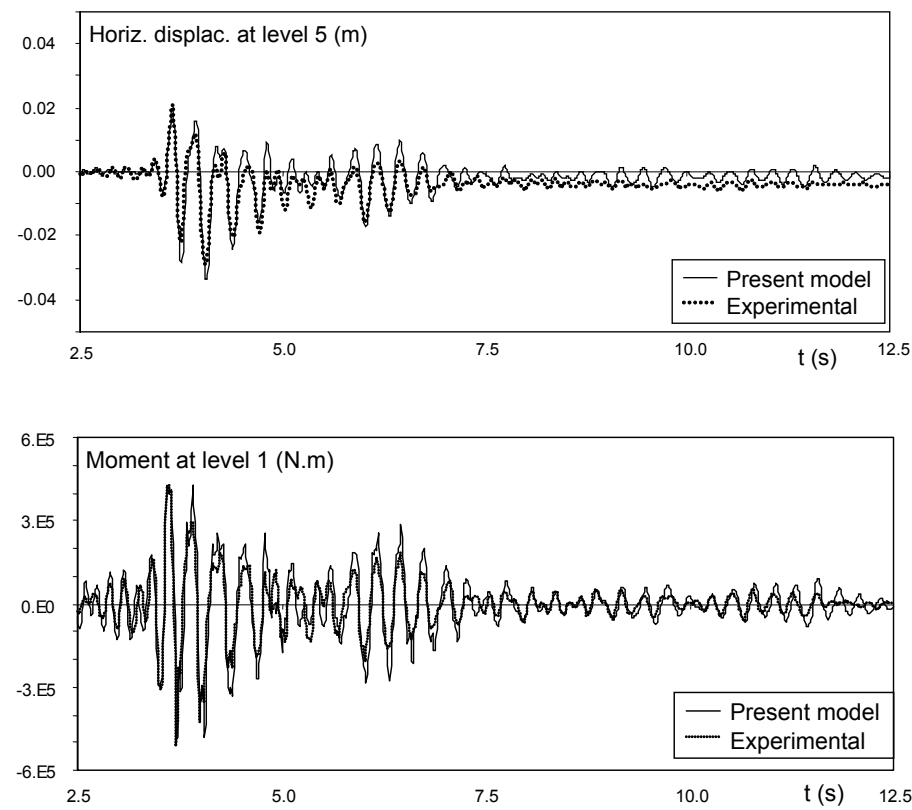

FIG. 7. Historical Results 

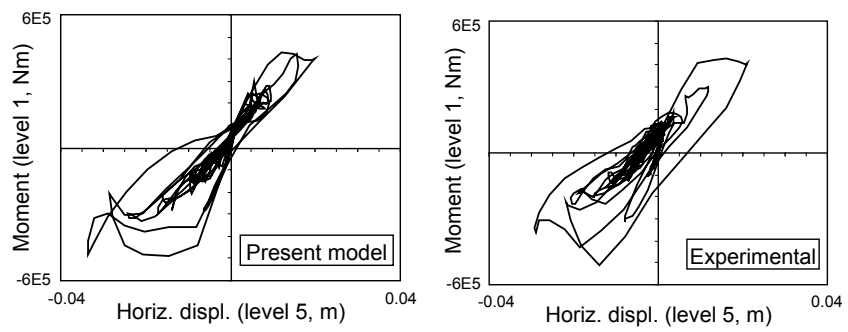

FIG. 8. Moment-Displacement Diagrams 


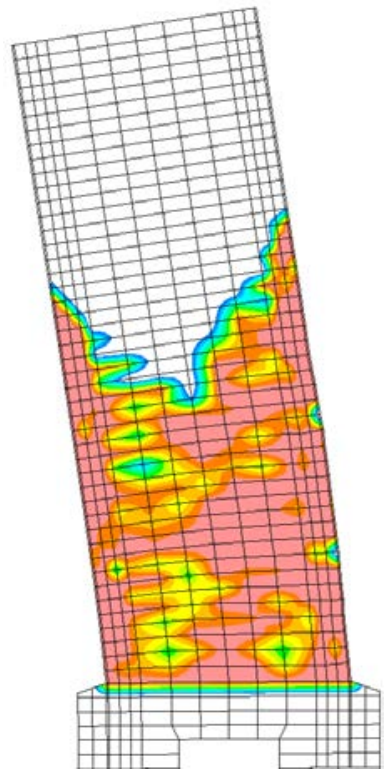

a) tensile damages

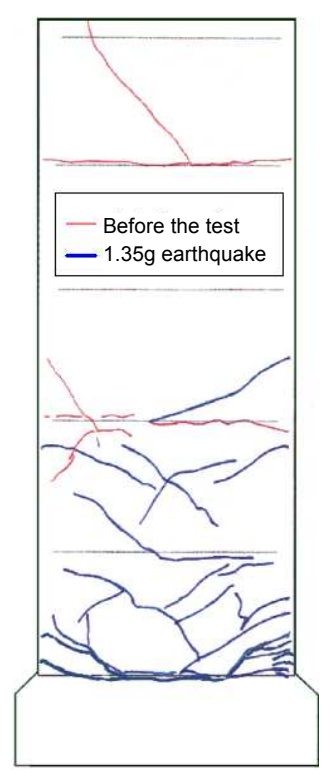

b) observed cracks

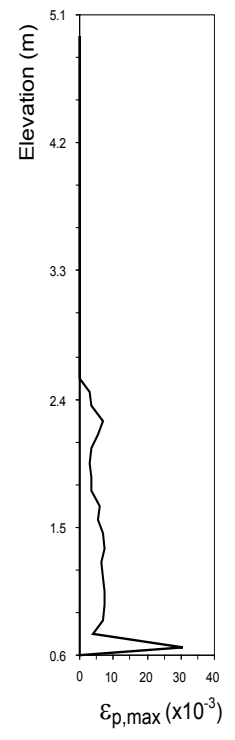

c) plastic strain

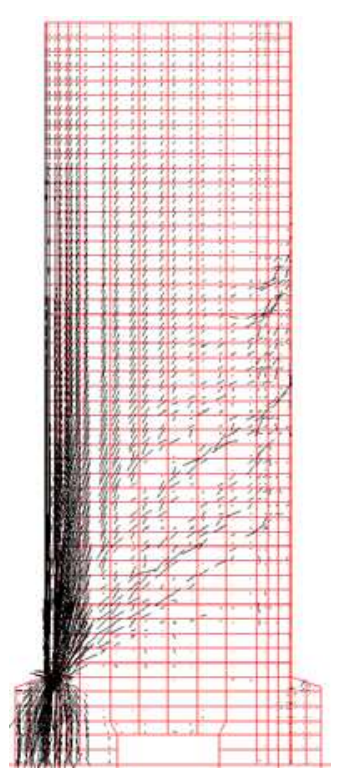

d) $\sigma^{-}$

(rightmost $\phi 8$ )

FIG. 9. Situation at the Peak of the $1.35 \mathrm{~g}$ Earthquake $(t=4 \mathrm{~s})$ 


\title{
Figure legends
}

FIG. 1. a) 2D Elastic Domain; b) Hardening/Softening Condition

FIG. 2. 1D Cyclic Performances of: $a$ ) Concrete; b) Steel Submodels

\author{
FIG. 3. Alto Lindoso Arch Dam
}

FIG. 4. Deformation and $d^{+}$During a $0.25 \mathrm{~g}$ Earthquake

$$
\left(d^{+}=1 \text { in gray; }=0 \text { in white }\right)
$$

FIG. 5. FE Meshes and Geometrical Data for the RC Wall

FIG. 6. a) 1.35g Accelerogram; b) Confined and Unconfined Concrete

FIG. 7. Historical Results

FIG. 8. Moment-Displacement Diagrams

FIG. 9. Situation at the Peak of the $1.35 \mathrm{~g}$ Earthquake $(t=4 \mathrm{~s})$ 